\title{
PLANETARY NEBULA BIRTH RATES IN THE GALAXY AND OTHER GALAXIES
}

\author{
MANUEL PEIMBERT \\ Instituto de Astronomía, UNAM, Apartado Postal 70-264, México 04510 D.F., México
}

\begin{abstract}
A review is presented of PN birth rates. The observational determinations for other galaxies are compared with theoretical predictions based on stellar evolution models. The galactic birth rate is derived for different distance scales and is compared with the predicted one from galactic models of the solar neighborhood and with those determined for other galaxies; to reach agreement between observations and predictions long distance scales to PN have to be adopted. The total number of PN is estimated for the Galaxy and other galaxies.
\end{abstract}

\section{Introduction}

Phillips (1989) presented an excellent review on the estimates of: the PN formation rate, $\dot{\rho}\left(\mathrm{pc}^{-3} \mathrm{yr}^{-1}\right)$, and the total number of $\mathrm{PN}$ in the Galaxy, $N_{T}$. Estimates of $N_{T}$ and $\dot{\rho}$ comprised a range of about two orders of magnitude. In this review I will try to estimate the constraints in the PN birth rate per unit luminosity, $\dot{\xi}\left(\mathrm{yr}^{-1} L_{\odot}^{-1}\right)$, and $N_{T}$ provided by stellar evolution models of a stellar ensemble (Renzini and Buzzoni 1986, Chiosi 1992) and by the seminal work of Jacoby and collaborators on the determinations of $\dot{\xi}$ and $N_{T}$ for extragalactic systems (Jacoby 1980, 1989; Jacoby et al. 1989, 1990a, 1990b; Ciardullo et al. 1989a, 1989b, 1991; Ciardullo and Jacoby 1992). An earlier account of some of these matters was presented by Peimbert (1990a).

\section{Birth rates}

The determinations of galactic birth rates of PN are important to: a) find out which is the fraction of the stars in the $0.8 \leq M_{i}\left(M_{\odot}\right) \leq 8$ range that undergo the PN phase, $\mathrm{b}$ ) to study the chemical enrichment of the interstellar medium, and c) to test the different statistical distance scales proposed for galactic PN.

The local PN birth rate is given by

$$
\dot{\rho}=\rho / \Delta t=\rho\langle v\rangle /\left(R_{f}-R_{i}\right),
$$

where $\rho$ is the density, in the solar vicinity, of PN with $R_{i}<R<R_{f}, \Delta t$ is the time needed for $R$ to increase from $R_{i}$ to $R_{f}$, and $\langle v\rangle$ is the average velocity of expansion from $R_{i}$ to $R_{f}$. Usually $\rho$ is given in $\mathrm{pc}^{-3}$ and $\Delta t$ in years; $R$ denotes the radius of ionized hydrogen.

In the optically thick (ionization bounded) phase $\langle v\rangle$ denotes the average velocity of the ionization front relative to the central star, $v_{i o n}$, while in the optically thin (matter bounded) phase $\langle v\rangle$ denotes the average velocity of expansion of matter, $v_{\text {exp }}$, 
given by the Doppler effect.

There are several sources of error associated with the use of equation (1) (see Phillips 1989, Peimbert 1990b). The main uncertainty is due to the adopted distance scale: since $\dot{\rho}$ is proportional to $d^{-4}$ the $\dot{\rho}$ estimates vary to a first approximation like $k^{-4}$ (see Table 3).

We will use the birth rate per unit luminosity, $\dot{\xi}$, instead of $\dot{\rho}$ because it is a more convenient quantity to compare with results for other galaxies and with model predictions for stellar ensembles. The galactic PN birth rate, $\dot{N}_{T}\left(\mathrm{yr}^{-1}\right)$, has been estimated by multiplying $\dot{\xi}$ by the bolometric luminosity of the Galaxy. The total number of PN has been derived by multiplying the galactic birth rate by the mean lifetime of a PN.

\section{Stellar death rate}

It is possible to compare $\dot{\xi}$ with the stellar death rate per solar bolometric luminosity, $\dot{S}$. Renzini and Buzzoni (1986) computed $\dot{S}_{b}$ values as a function of time from models with a single burst of star formation, without subsequent star formation or star accretion, and three widely different initial mass functions, $\mathrm{IMF}$, independent of time and chemical composition. Their assumed IMF by number are given by: $\phi\left(M_{i}\right)=A M_{i}^{-\alpha}$, with $\alpha$ equal to $1.5,2.35$ and 3.5 for $M_{i} \geq 0.57 M_{\odot}$ and $\alpha=2.35$ for $M_{i}<0.57 M_{\odot}$ for the three IMF.

Peimbert (1990a) has shown that to a very good approximation $\dot{S}_{b}\left(t_{1}\right)$, where $t_{1}$ is the age of the system, corresponds to the stellar death rate per unit luminosity for systems with a constant rate of star formation and for systems with a decreasing rate of star formation. This result is very powerful and implies that for galaxies where star formation started more than $10^{9}$ years ago and that are not suffering from an extremely violent burst of star formation, $\dot{S}\left(10^{-12} \mathrm{yr}^{-1} L_{\odot}^{-1}\right)$ should be in the 18 to 22 range; moreover it also implies that if in these systems all the stars in the $0.8 \leq M\left(M_{\odot}\right) \leq 8$ range go through the PN phase then $\dot{\xi}=\dot{S}$. Chiosi (1992) from an independent study of the stellar death rate obtains very similar results to those of Renzini and Buzzoni (1986).

\section{Extragalactic systems}

Table 1 presents the absolute bolometric magnitudes of 17 galactic systems. Also Table 1 presents the $\dot{\xi}$ and $N_{T}$ values based on the following assumptions: a) the PN luminosity function established by Jacoby (1989), Jacoby et al. (1989) and Ciardullo et al. (1989b) applies to all the systems and b) the lifetimes of the $\mathrm{PN}$ are of 25,000 years.

In Figures 1a and $1 \mathrm{~b}$ we present the $(B-V)_{0}$ versus $\dot{\xi}$ and $M_{b o l}$ versus $\dot{\xi}$ diagrams respectively. In these figures NGC 205 and NGC 185 were not plotted due to the large estimated errors. The errors quoted correspond to the square root over the total number of detected PN but they do not include errors in the estimated lifetimes nor in the PNLF. The $\dot{\xi}$ values for the SMC and the LMC are smaller than those presented by Peimbert (1990a) mainly due to the study made by Boroson and Liebert (1989) who found that a considerable fraction of the PN candidates in the list by Jacoby (1980) were not PN.

The decrease of $\dot{\xi}$ with $(B-V)_{0}$ and with increasing luminosity has been discussed by Peimbert (1990a) and by Ciardullo et al. (1991).

For all the galaxies in Table 1 a value of $\dot{S}$ in the range of $15 \leq 10^{-12} \mathrm{yr}^{-1} L_{\odot}^{-1} \leq$ 22 is predicted; slightly higher $\dot{S}$ values for the more luminous galaxies are expected due to their older stellar population (see Peimbert 1990a). Therefore the decrease of $\dot{\xi}$ with $(B-V)_{0}$ and with $M_{b o l}$ is not due to a decrease in $\dot{S}$. 
TABLE 1. Distance moduli, absolute bolometric magnitudes, birth rates and total number of PN.

\begin{tabular}{lccccc}
\hline Object & $(m-M)_{0}$ & $M_{b o l}$ & $\begin{array}{c}\dot{\xi} \\
\left(10^{-12} \mathrm{yr}^{-1} L_{\odot}^{-1}\right)\end{array}$ & $\begin{array}{c}N_{T} \\
\left(10^{3}\right)\end{array}$ & References \\
& & & & \\
\hline NGC 4472 & 30.71 & -23.29 & $2.7 \pm 0.6$ & $11.1 \pm 2.5$ & $1,2,3,4,5$ \\
NGC 4486 & 30.81 & -23.08 & $3.4 \pm 0.6$ & $11.5 \pm 2.0$ & $1,3,4,5,6$ \\
NGC 4649 & 30.76 & -22.85 & $2.6 \pm 0.8$ & $7.1 \pm 2.2$ & $1,2,3,4,5$ \\
NGC 4406 & 30.98 & -22.78 & $5.4 \pm 0.8$ & $13.9 \pm 2.1$ & $1,2,3,4,5$ \\
NGC 4374 & 30.98 & -22.60 & $6.8 \pm 1.3$ & $14.8 \pm 2.8$ & $1,2,3,4,5$ \\
& & & & & \\
NGC 4382 & 30.79 & -22.31 & $8.0 \pm 1.2$ & $13.3 \pm 2.0$ & $1,3,4,5,6$ \\
M31 & 24.26 & -21.96 & $6.6 \pm 1.2$ & $8.0 \pm 1.5$ & $5,7,8,9$ \\
M81 & 27.72 & -21.88 & $8.4 \pm 1.8$ & $9.4 \pm 2.0$ & $5,10,11$ \\
NGC 3379 & 29.96 & -21.57 & $8.5 \pm 1.7$ & $7.2 \pm 1.4$ & $3,5,8,12$ \\
NGC 1023 & 29.97 & -21.51 & $9.1 \pm 1.5$ & $7.3 \pm 1.2$ & $3,8,13,14,15$ \\
& & & & & \\
NGC 3384 & 30.03 & -21.25 & $15.0 \pm 3.0$ & $9.4 \pm 1.9$ & $3,5,8,12$ \\
NGC 3377 & 30.07 & -20.69 & $15.0 \pm 3.0$ & $5.6 \pm 1.4$ & $3,5,8,12$ \\
LMC & 18.44 & -19.03 & $8.1 \pm 1.8$ & $0.64 \pm 0.14$ & $8,15,16,17,18,19,20$ \\
SMC & 18.76 & -17.20 & $7.7 \pm 2.3$ & $0.12 \pm 0.04$ & $8,15,16,17,18,19,21$ \\
M32 & 24.26 & -17.14 & $10.1 \pm 2.5$ & $0.14 \pm 0.04$ & $5,7,8$ \\
& & & & & \\
NGC 205 & 24.26 & -16.86 & $23.5 \pm 6.8$ & $0.26 \pm 0.08$ & $7,8,15,22$ \\
NGC 185 & 23.78 & -15.89 & $14.5 \pm 7.3$ & $0.07 \pm 0.04$ & $7,8,15,23$ \\
\hline
\end{tabular}

1) Burstein and Heiles 1984, 2) Poulain 1988, 3) Sandage and Tammann 1981, 4) Jacoby et al. 1990a, 5) Peimbert 1990a, 6) Michard 1982, 7) Ciardullo et al. 1989b, 8) de Vaucouleurs et al. 1976, 9) Freeman 1970, 10) Brandt et al. 1972, 11) Jacoby et al. 1989, 12) Ciardullo et al. 1989a, 13) Frogel et al. 1978, 14) Ciardullo et al. 1991, 15) This work, 16) Hindman 1967, 17) Kruit 1990, 18) Bothun and Thompson 1988, 19) Jacoby et al. 1990b, 20) Boroson and Liebert 1989, 21) Feast 1988, 22) Ciardullo and Jacoby 1992, 23) Saha and Hoessel 1990.

Most of the PNLF used for the determinations of $\dot{\xi}$ in Table 1 are complete only at the high luminosity end; the completeness limit of the observations extends only from 0.8 to 2.5 mag below the bright end cutoff. The total number of PN is obtained by adjusting the observed upper end of the PNLF with the PNLF derived by Jacoby and collaborators. Since the PNLF spans about 8 magnitudes (Ciardullo et al. 1989b), the $\dot{\xi}$ values represent the upper end of the PNLF. Therefore strictly speaking, it can only be said that the number of bright $\mathrm{PN}$ decreases with increasing luminosity of the galaxy for $(B-V)_{0}>0.90$ or $M_{b o l}<-22.0$

The decrease in the number of bright $\mathrm{PN}$ with the increase of $(B-V)_{0}$ and luminosity could be due to: a) an age effect in galaxies with stellar populations of different ages (Peimbert 1990a) or b) to a spread in the masses of the central stars of PN, $M_{c}$, produced by stars with similar initial masses in the main sequence (Ciardullo et al. 1991). In both cases larger ages will produce fainter and fewer PN due to the following reasons: a) lower luminosity of the central star, b) smaller mass of the shell and c) longer stellar evolutionary times that might prevent the star to become hot enough to ionize the nebula before it has dissipated. 


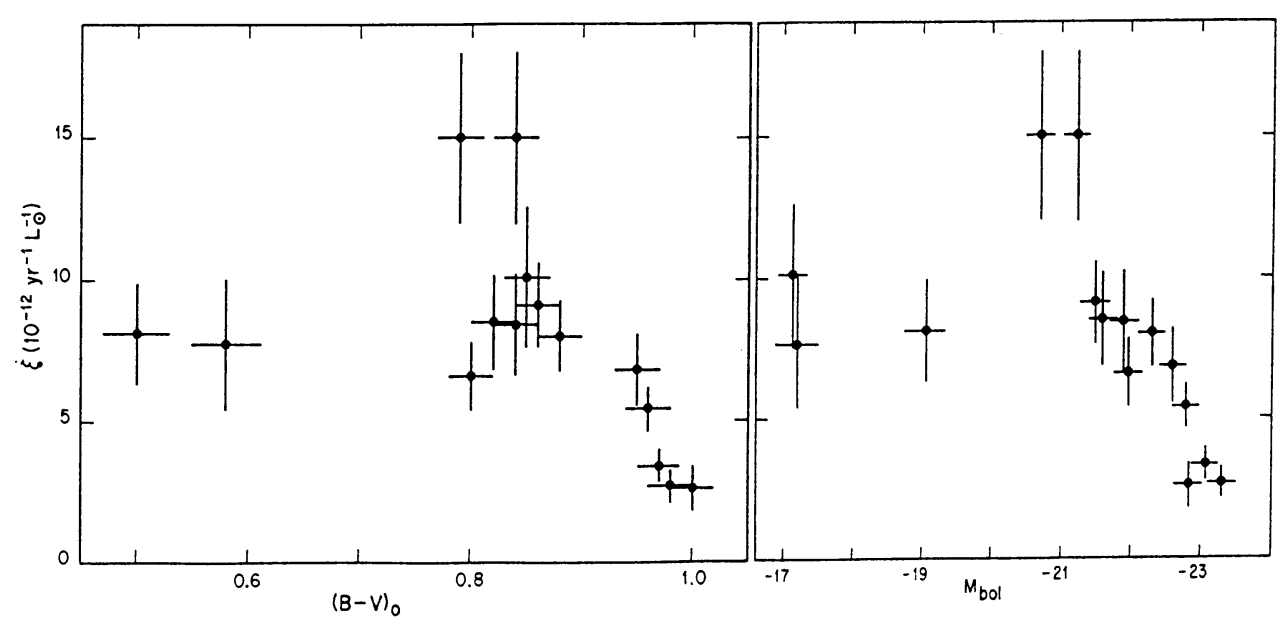

Figure 1. a) PN birth rate per solar luminosity, $\dot{\xi}$, versus intrinsic color index, $(B-V)_{0}$, for a group of fifteen galaxies. b) $\dot{\xi}$ versus bolometric magnitude, $M_{b o l}$.

\section{The Galaxy}

\subsection{GALACTIC MODELS}

To derive $\dot{\xi}$ and $N_{T}$ for the Galaxy we need to know the luminosity of the solar neighborhood and the $M_{b o l}$ for the whole system. Table 2 presents galactic properties based on three different sources, they are very similar; in what follows we will adopt $M_{b o l}=-21.8$ for the Galaxy.

Other authors have determined $N_{T}$ based on the specific number of PN per unit mass (e.g. Alloin et al. 1976, Phillips 1989). We consider the determinations of $N_{T}$ based on the number of PN per unit luminosity to be more accurate than those derived from the number of PN per unit mass. All the $N_{T}$ values presented in this review are based on the luminosity of the system involved.

TABLE 2. Galactic properties

\begin{tabular}{|c|c|c|c|c|c|c|c|}
\hline \multicolumn{4}{|c|}{ Solar Neighborhood } & \multicolumn{3}{|c|}{ Whole Galaxy } & \multirow{2}{*}{$\begin{array}{l}\text { Refer- } \\
\text { ences }\end{array}$} \\
\hline $\begin{array}{c}\mathrm{L} \\
\left(L_{\odot \mathrm{pc}}{ }^{-3}\right)\end{array}$ & $\begin{array}{c}\mathrm{L}_{v} \\
\left(L_{\odot} \mathrm{pc}^{-3}\right)\end{array}$ & $\begin{array}{c}\mu_{v} \\
\left(L_{\odot p c^{-2}}\right)\end{array}$ & B.C. & $\mathrm{B}-\mathrm{V}$ & $\mathrm{M}_{v}$ & $\mathrm{M}_{b o l}$ & \\
\hline $1.15 \times 10^{-1}$ & $5.34 \times 10^{-2}$ & $\ldots$ & -0.84 & $\ldots$ & -20.5 & $-21.3:$ & 1 \\
\hline$\ldots$ & $\ldots$ & $\ldots$ & $\ldots$ & $0.53 \pm 0.05$ & -20.6 & $-21.4:$ & 2 \\
\hline$\ldots$ & $\ldots$ & $22.2 \pm 2.8$ & $\ldots$ & $0.85 \pm 0.15$ & -21.15 & $-21.95:$ & 3,4 \\
\hline
\end{tabular}

1) Allen 1973, 2) de Vaucouleurs and Pence 1978, 3) Kruit 1986, 4) Kruit 1990. 
TABLE 3. A comparison of distance scales for PN with $0.10 \leq R(\mathrm{pc}) \leq 0.30$.

\begin{tabular}{lcl}
\hline$k=d / d_{\text {Seaton }}$ & $\mathrm{M}(\mathrm{rms})$ & $\begin{array}{l}\text { Scale } \\
\left(M_{\odot}\right)\end{array}$ \\
\hline 1.43 & 0.50 & Cudworth (1974) \\
1.41 & 0.49 & Kingsburgh and Barlow (1992) \\
1.41 & $\ldots$ & Mallik and Peimbert (1988) \\
1.40 & 0.47 & Schneider and Terzian (1983) \\
1.30 & 0.39 & Weidemann (1977) \\
1.06 & $\ldots$. & Maciel and Pottasch (1980) \\
1.00 & 0.20 & Seaton (1968) \\
1.00 & 0.20 & Cahn and Kaler (1971) \\
1.00 & 0.20 & Milne and Aller (1975) \\
0.86 & 0.14 & Daub (1982) \\
\hline
\end{tabular}

\subsection{DISTANCE SCALES}

The density of PN in the solar neighborhood depends on the distance scale to the fourth power, see equation (1). The distance scale is still a controversial issue and widely different distance scales are used by different authors. To compare different distance scales it is possible to introduce a relative scale factor, $k$, with the normalization $k=1$ for Seaton's (1968) distance scale. In Table 3 we present a comparison in the $0.10 \leq R(\mathrm{pc}) \leq 0.30$ range for some of the most frequently used distance scales. For optically thin PN samples $k \propto M(\mathrm{rms})^{2 / 5}$, where $M(\mathrm{rms})$ is the envelope mass derived from the root mean square density. For optically thick PN samples $k$ increases with $R$ and an average value for the considered range is presented in Table 3 . If objects smaller than $0.1 \mathrm{pc}$ are considered the spread in $k$ values is even larger (Gathier 1987). In this comparison the $M(\mathrm{rms})$ values have been computed under the assumptions that $\mathrm{N}(\mathrm{He}) / \mathrm{N}(\mathrm{H})=0.11$ and $\mathrm{N}\left(\mathrm{He}^{+}\right) / \mathrm{N}\left(\mathrm{H}^{+}\right)$ $+2 \mathrm{~N}\left(\mathrm{He}^{++}\right) / \mathrm{N}\left(\mathrm{H}^{+}\right)=0.13$.

\subsection{BIRTH RATES AND TOTAL NUMBER OF PN}

Table 4 presents four determinations of $\dot{\xi}$. For the first determination we took the surface density of PN by Mallik (1991), $\sigma=5.8 \pm 1.2 \mathrm{kpc}^{-2}$, the $\mu_{v}$ value in Table 2 , a lifetime of 25,000 years and a B.C. of $0.84 \mathrm{mag}$. For the second value we took the volume density of PN by Mallik, $\rho=19 \pm 4 \mathrm{kpc}^{-3}$, the $L$ value in Table 2 and a lifetime of 25,000 years. The errors come from the PN densities. The difference between the first two determinations is mainly due to the different scale heights of PN and stellar luminosity in the solar neighborhood (see $\S 5.4$ ). The third $\dot{\xi}$ value was derived from Figure 1a assuming a $(B-V)_{0}=0.85$ for the Galaxy and the last $\dot{\xi}$ value from Figure $1 \mathrm{~b}$ and assuming $M_{b o l}=-21.8$ for the Galaxy (see Table 2).

Table 5 presents $\dot{\xi}$ determinations derived from $\rho$ values by different authors, that were obtained based on different samples and different distance scales, and the $\mathrm{L}$ value by Allen (1973) for the solar neighborhood presented in Table 2. We have assumed a lifetime of 25,000 years for the PN, with the exception of the determination by Ishida and Weinberger (1987) for which we used a lifetime of 40,000 years, given by them, because they are considering larger and fainter nebulae than the other authors. For all the $N_{T}$ determinations we assumed a lifetime of 25,000 years for the PN and an $M_{b o l}=-21.8$ for the Galaxy. Most of the differences in Table 5 are due to the distance scale adopted. 
TABLE 4. Galactic PN birth rate

\begin{tabular}{cll}
\hline $\begin{array}{c}\dot{\xi} \\
\left(10^{-12} \mathrm{y} \mathrm{r}^{-1} L_{\odot}^{-1}\right)\end{array}$ & \multicolumn{1}{c}{ Method } & References \\
\hline $4.8 \pm 1.0$ & $\begin{array}{l}\text { Surface density of PN and surface brightness } \\
\text { at the solar galactocentric distance }\end{array}$ & $1,2,3,4$ \\
$6.6 \pm 1.4$ & $\begin{array}{l}\text { Local density of PN and solar neighborhood } \\
\text { luminosity function }\end{array}$ & $1,2,3,5$ \\
$9.0 \pm 3$ & $(B-V)_{0}$ versus $\dot{\xi}$ (Fig. 1a and Table 2) & 3 \\
$9.0 \pm 3$ & $M_{b o l}$ versus $\dot{\xi}$ (Fig. 1b and Table 2) & 3 \\
\hline
\end{tabular}

1) Mallik and Peimbert 1988, 2) Mallik 1991, 3) this work, 4) Kruit 1986, 5) Allen 1973.

TABLE 5. WD and PN birth rates in the solar neighborhood and the total number of PN in the Galaxy

\begin{tabular}{cccll}
\hline $\begin{array}{c}\dot{\xi} \\
\left(10^{-12} \mathrm{yr}^{-1} L_{\odot}^{-1}\right)\end{array}$ & & $\begin{array}{c}N_{T}(\mathrm{PN}) \\
\left(10^{3}\right)\end{array}$ & $k$ & References \\
\hline $5.4 \pm 1.1$ & WD & $\ldots$ & $\ldots$ & 1 \\
$6.3 \pm 2.2$ & WD & $\cdots$ & $\cdots$ & 2 \\
$6.6 \pm 1.4$ & $\mathrm{PN}$ & 6.9 & 1.41 & 3 \\
$10.1 \pm 2.6$ & $\mathrm{PN}$ & 10.5 & 1.43 & $4,5,6$ \\
$12.2 \pm 2.2$ & $\mathrm{PN}$ & 12.7 & 1.30 & 7 \\
$16.6 \pm 2.2$ & $\mathrm{PN}$ & 17.3 & $\cdots$ & 8 \\
16.7 & $\mathrm{PN}$ & 17.4 & $\cdots$ & 9 \\
20.0 & WD & $\cdots$ & $\cdots$ & 10 \\
$42.3 \pm 11$ & $\mathrm{PN}$ & 44.1 & 1.00 & $5,6,11$ \\
$43.5 \pm 17$ & $\mathrm{PN}$ & 45.3 & 0.86 & 6,12 \\
69.6 & $\mathrm{PN}$ & 72.6 & $\cdots$ & 13 \\
\hline
\end{tabular}

1) Fleming et al. 1986, 2) Downes 1986, 3) Mallik 1991, 4) Cudworth 1974, 5) Alloin et al. 1976, 6) Peimbert 1990a, this work, 7) Weidemann 1977, 8) Phillips 1989, 9) Acker 1978, 10) Weidemann 1991, 11) Cahn and Kaler 1971, 12) Daub 1982, 13) Ishida and Weinberger 1987.

\subsection{SCALE HEIGHTS}

To compare the $\dot{S}$ values from galactic evolution models with the observed $\dot{\xi}$ values we need to know if all the stars go through the PN phase, this can be tested by comparing the scale heights of the PN and of the stellar luminosity of the solar neighborhood.

The scale heights derived by different authors are: 90 pc (Cahn and Kaler 1971), $100 \mathrm{pc}$ (Ishida and Weinberger 1987), $115 \mathrm{pc}$ (Cahn and Wyatt 1976), $125 \mathrm{pc}$ (Daub 1982), 130 pc (Amnuel et al. 1984) and 153 pc (Mallik 1991). In general the larger the distance scale adopted the larger the derived scale height. These scale heights can be compared with those derived for the different populations in the solar neighborhood. The 
model of Kruit (1986) has a scale height of $80 \mathrm{pc}$ for population I stars with $L_{v}(z=0)=$ $39.5 \%$ and a scale height of 325 pc for old dwarfs and disk giants with $L_{v}(z=0)=60.5 \%$. From the model of Kruit (1986) and Table 2 I assume that the luminosity distribution in the solar neighborhood can be approximated by

$$
L(z)=1.15 \times 10^{-1}\left(0.40 e^{-z / 80}+0.60 e^{-z / 325}\right) L_{\odot} \mathrm{pc}^{-3},
$$

where $z$ is the distance to the plane in pc, the first term corresponds to the population I component and the second to the old disk population. The scale height of $L(z)$ is approximately $227 \mathrm{pc}$. The population I component with $M<8 M_{\odot}$ has $\dot{S}=18 \times$ $10^{-12} \mathrm{yr}^{-1} L_{\odot}^{-1}$ while the old disk component has $\dot{S}=21 \times 10^{-12} \mathrm{yr}^{-1} L_{\odot}^{-1}$ (Renzini and Buzzoni 1986). By assuming that all the stars of the population I component with $M<$ $8 M_{\odot}$ produce PN and that only a fraction of the old disk stars produce PN we have computed Table 6 . From this table we obtain that approximately $28 \%$ of the old disk stars go through the PN stage for the scale height derived by Mallik (1991). In Figure 2 the region to the left of the model is permitted and implies that long distance scales with $k \geq 1.3$ and $h \geq 140 \mathrm{pc}$ should be used. In Table 7 we present $\dot{\xi}, h, N_{T}$ and $\dot{N}_{T}$ as a function of $k$ based on the local density of PN determined by Mallik.

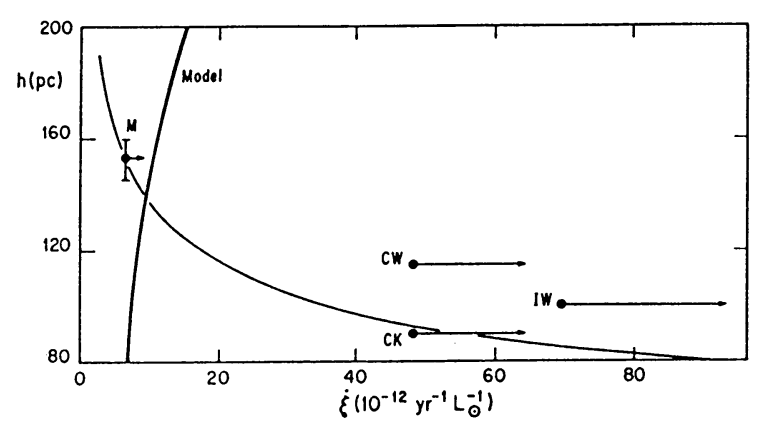

Figure 2. $\dot{\xi}$ versus scale height, $h$, for the solar neighborhood. The four points correspond to: Mallik $(k=1.41)$, Ishida and Weinberger, Cahn and Kaler $(k=$ $1)$ and Cahn and Wyatt $(k=1)$. The model values come from Table 6 . The other curve indicates the values derived from Mallik (1991) assuming a variable $k$ factor. The arrows indicate the growth in $\dot{\xi}$ under the assumption that the lifetime of $\mathrm{PN}$ is of 18,750 years instead of 25,000 .

TABLE 6. Birth rate for $z=0 \mathrm{pc}$ as a function of the PN scale height.

\begin{tabular}{ccc}
\hline $\begin{array}{c}\dot{\xi} \\
\left(10^{-12} \mathrm{yr}^{-1} L_{\odot}^{-1}\right)\end{array}$ & $\begin{array}{c}\mathrm{h} \\
(\mathrm{pc})\end{array}$ & $\begin{array}{c}\text { \% of old disk stars } \\
\text { that become PN }\end{array}$ \\
\hline 7.2 & 80 & 0 \\
7.9 & 100 & 6 \\
9.1 & 125 & 15 \\
10.7 & 150 & 27 \\
12.5 & 175 & 42 \\
15.3 & 200 & 64 \\
19.4 & 225 & 97 \\
19.8 & 227 & 100 \\
\hline
\end{tabular}


TABLE 7. $\dot{\xi}, \mathrm{h}, \mathrm{N}_{T}$ and $\dot{N}_{T}$ as a function of the galactic distance scale, $k$, based on the local volume density of PN determined by Mallik (1991). Hubble parameter based on the consistency of the galactic and extragalactic PNLF with $k=1.20$ and $H_{0}=74 \mathrm{~km} \mathrm{~s}^{-1}$ $\mathrm{Mpc}^{-1}$ (Méndez et al. 1992a, 1992b, Jacoby and Ciardullo 1992).

\begin{tabular}{lccccc}
\hline$k$ & $\begin{array}{c}\dot{\xi} \\
\left(10^{-12} \mathrm{yr}^{-1} L_{\odot}^{-1}\right)\end{array}$ & $\begin{array}{c}N_{T} \\
(\mathrm{pc})\end{array}$ & $\begin{array}{c}\dot{N}_{T} \\
\left(10^{3}\right)\end{array}$ & $\begin{array}{c}\mathrm{H}_{0} \\
\left(\mathrm{yr}^{-1}\right)\end{array}$ & $\left(\mathrm{km} \mathrm{s}^{-1} \mathrm{Mpc}^{-1}\right)$ \\
\hline 0.8 & 64.5 & 87 & 67 & 2.7 & 111 \\
1.00 & 27.2 & 109 & 28 & 1.13 & 89 \\
1.20 & 12.7 & 130 & 13.2 & 0.53 & 74 \\
1.40 & 7.1 & 152 & 7.4 & 0.30 & 63 \\
1.50 & 5.4 & 163 & 5.6 & 0.22 & 59 \\
1.75 & 2.9 & 190 & 3.0 & 0.12 & 51 \\
2.00 & 1.7 & 217 & 1.8 & 0.07 & 44 \\
\hline
\end{tabular}

\section{Optical thickness}

The assumption of 25,000 years for the $\mathrm{PN}$ lifetime, combined with a velocity of expansion of $25 \mathrm{~km} \mathrm{~s}^{-1}$ (Phillips 1989), imply a limiting size of $R=0.64 \mathrm{pc}$ for optically thin PN. If we assume that the samples are made of optically thin PN with $R<0.64 \mathrm{pc}$, then the $\dot{\xi}$ values in Tables $1,4,5$ and 7 are correct. On the other hand if we assume that $R_{\max }=0.64 \mathrm{pc}$ and that during part of their lifetimes the PN are optically thin and the rest optically thick, the lifetimes become smaller because during the optically thick phase $v_{i o n}$ should be used instead of $v_{\text {exp }}$.

From the distance independent sample by Mallik and Peimbert (1988) it follows that most PN in the solar neighborhood are optically thick, in at least some directions, a similar result was obtained by Gathier (1987). A complementary result was obtained by Méndez et al. (1992a) who find that most PN in their sample are optically thin in at least some directions. Consequently a large fraction of PN are optically thick in some directions and thin in others. Marten (1992) finds for an optically thick model of PN that $v_{i o n} / v_{\text {exp }} \approx 1.5$. By assuming that during part of their evolution PN are optically thick, then their lifetimes will get reduced and their $\dot{\xi}$ values will be increased by $v_{i o n} / v_{e x p}$; this effect will affect the $\dot{\xi}$ values in Tables $1,4,5$, and 7 but obviously will not affect the $N_{T}$ values in all tables nor the $\dot{\xi}$ values in Table 6 . In Figure 2 the arrows represent an increase of 1.33 in the $\dot{\xi}$ values.

PN with $R>0.64$ pc present several problems and I consider that it is a good idea to avoid them in the determination of $\dot{\xi}$ : a) the PN samples suffer from severe incompleteness of intrinsically large PN due to their faintness, b) their expansion could be decelerated by the interstellar medium (Ishida and Weinberger 1987), c) in the late stages of evolution PN could become optically thick again, and if the number of ionizing photons decreases rapidly with time the ionized size could also decrease with time.

\section{Discussion}

By comparing the distances of Cudworth (1974) and Méndez et al. (1992a) I find that $k=1.20 \pm 0.2$ for the latter sample, the large error comes from the small number of PN involved. The Méndez et al. sample has a selection effect in favor of high latitude 
PN, which presumably have on the average fainter stars than the samples used by other authors; notice however that Cudworth (1974) does not find a significant difference in the distance scales for B and C nebulae. Méndez et al. (1992b) find that the galactic PNLF derived from the Méndez et al. (1992a) sample is similar to the extragalactic PNLF derived by Jacoby and collaborators. If the galactic PNLF derived from the sample of Méndez et al. (1992a) agrees with the extragalactic one, the $H_{0}$ value of $74 \mathrm{~km} \mathrm{~s}^{-1} \mathrm{Mpc}^{-1}$ derived by Jacoby and Ciardullo (1992) corresponds to $k=1.2$; the error in this equivalence is of a factor of $\sim 1.2$. In Table 7 we present the $H_{0}$ values for different $k$ values.

The decrease of $\dot{\xi}$ with increasing $M_{b o l}$ for galaxies brighter than $M_{b o l}=-\mathbf{2 2 . 0}$ or redder than $(B-V)_{0}=0.90$ could be due to a smaller fraction of the older stars producing $\mathrm{PN}$ in agreement with the scale height determination for the Galaxy where we find that only $\sim 28 \%$ of the old disk population objects go through the PN stage.

The $\dot{\xi}$ determinations derived from: a) the $(B-V)_{0}$ and $M_{b o l}$ values for the Galaxy (Figure 1 and Table 2) for $v_{i o n} / v_{\text {exp }}=1.3, \mathrm{~b}$ ) the distance scales by Cudworth (1974) and Mallik and Peimbert (1988) (Table 5) for $v_{\text {ion }} / v_{\text {exp }}=1.3$ and c) the scale height determinations by Mallik (1991) (Table 6), imply that $\langle\dot{\xi}\rangle=(11 \pm 3) \times 10^{-12} \mathrm{yr}^{-1} L_{\odot}^{-1}$ for the Galaxy. This value compared with the results by Renzini and Buzzoni (1986) implies that about half of the IMS go through the PN phase.

Similarly from the same sources it is found that $N_{T}=9000 \pm 3000$. From a) and c) above it is found that $k=1.4 \pm 0.1$; where the upper limit comes mainly from the $\dot{\xi}$ extragalactic results, and the lower limit from the stellar death rate predictions and the PN scale height.

From the $\langle\dot{\xi}\rangle$ value for the Galaxy it is found that $\dot{N}_{T}(\mathrm{PN})=0.46 \mathrm{yr}^{-1}$. Alternatively from Renzini and Buzzoni (1986) if all the stars go through the PN phase then $\dot{N}_{T}=0.83 \mathrm{yr}^{-1}$. These numbers can be compared with the white dwarf formation rate: $N(\mathrm{WD})=0.25 \mathrm{yr}^{-1}$ (Fleming et al. 1986, Downes 1986), $\dot{N}(\mathrm{WD})=0.83 \mathrm{yr}^{-1}$ (Weidemann 1991); and the $\mathrm{OH} / \mathrm{IR}$ star formation rate $\dot{N}(\mathrm{OH} / \mathrm{IR}) \sim 0.9 \mathrm{yr}^{-1}$ (Herman and Habing 1985).

It is a pleasure to acknowledge several fruitful discussions with G.H. Jacoby, D.C.V. Mallik and R. Méndez.

\section{References}

Acker, A. 1978, Astr. and Ap. Suppl., 33, 367.

Allen, C.W. 1973, Astrophysical Quantities, (London: Athlone).

Alloin, D., Cruz-González, C., and Peimbert, M. 1976, Ap. J., 205, 74.

Amnuel, P.R., Guseinov, O.H., Novruzova, H.I., Rustamov, Yu. S. 1984, Ap. Sp. Sci., $107,19$.

Boroson, T.A. and Liebert, J. 1989, Ap. J., 339, 844.

Bothun, G.D. and Thompson, I.B. 1988, A.J., 96, 877.

Brandt, J.C., Kalinowski, J.K., and Roosen, R.G. 1972, Ap. J. Suppl., 24, 421.

Burstein, D., and Heiles, C. 1984, Ap. J. Suppl., 54, 33.

Cahn, J.H. and Kaler, J.B. 1971, Ap. J. Suppl., 22, 319.

Cahn, J.H. and Wyatt, S.P. 1976, Ap. J., 210, 508.

Chiosi, C. 1992 , private communication.

Ciardullo, R. and Jacoby, G.H. 1992, Ap. J., in press.

Ciardullo, R., Jacoby, G.H., and Ford, H.C. 1989a, Ap. J., 344, 715.

Ciardullo, R., Jacoby, G.H., Ford, H.C., and Neill, J.D. 1989b, Ap. J., 339, 53.

Ciardullo, R., Jacoby, G.H., and Harris, W.E. 1991, Ap. J., 383, 487. 
Cudworth, K.M. 1974, A.J., 79, 1384.

Daub, C.T. 1982, Ap. J., 260, 612.

de Vaucouleurs, G., de Vaucouleurs, A., and Corwin, H.G. Jr. 1976, Second Reference Catalogue of Bright Galaxies, (Austin: University of Texas Press).

de Vaucouleurs, G. and Pence, W.D. 1978, A.J., 84, 1163.

Downes, R.A. 1986, Ap. J. Suppl., 61, 569.

Feast, M.W. 1988, in The Extragalactic Distance Scale, A.S.P. Conference Series No. 4, ed. S. van den Bergh and C.J. Pritchet (Provo: Brigham Young University Press), p. 9.

Fleming, T.A., Liebert, J., and Green, R.F. 1986, Ap. J.,, 308, 176.

Freeman, K.C. 1970, Ap. J., 160, 811.

Frogel, J.A., Persson, S.E., Aaronson, M. and Matthews, K. 1978, Ap. J., 220, 75.

Gathier, R. 1987, Astr. and Ap. Suppl., 71, 245.

Herman, J. and Habing, H.J. 1985, Physics Reports, 124, No. 4, p. 255.

Hindman, J.V. 1967, Australian J. Phys., 20, 147.

Ishida, K. and Weinberger, R. 1987, Astr. and Ap., 178, 227.

Jacoby, G.H. 1980, Ap. J. Suppl., 42, 1.

Jacoby, G.H. 1989, Ap. J., 339, 39.

Jacoby, G.H. and Ciardullo, R. 1992, this Symposium.

Jacoby, G.H., Ciardullo, R., and Ford, H. 1990a, Ap. J., 356, 332.

Jacoby, G.H., Ciardullo, R., Ford, H.C., and Booth, J. 1989, Ap. J., 344, 704.

Jacoby, G.H., Walker, A.R. and Ciardullo, R. 1990b, Ap. J., 356, 332.

Kingsburgh, R.L. and Barlow, M.J. 1992, MNRAS, 257, 317.

Kruit, P.C. van der 1986, Astr. and Ap., 157, 230.

Kruit, P.C. van der 1990, in The Milky Way as a Galaxy, eds. G.F. Gilmore, I.R. King and P.C. van der Kruit (University Science Books, California) p. 331.

Maciel, W.J. and Pottasch, S.R. 1980, Astr. and Ap., 88, 1.

Mallik, D.C.V. 1991, Proc. Astron. Soc. Australia, 9, 15.

Mallik, D.C.V. and Peimbert, M. 1988, Rev. Mexicana Astron. Astrof., 16, 111.

Marten, H. 1992, this Symposium.

Méndez, R.H., Kudritzki, R.P. and Herrero, A. 1992a, Astr. and Ap., 260, 329.

Méndez, R.H., Kudritzki, R.P., Ciardullo, R. and Jacoby, G.H. 1992b, in preparation.

Michard, R. 1982, Astr. and Ap. Suppl., 49, 591.

Milne, D.K. and Aller, L.H. 1975, Astr. and Ap., 38, 183.

Peimbert, M. 1990a, Rev. Mexicana Astron. Astrof., 20, 119.

Peimbert, M. 1990b, Reports on Progress in Physics, 53, 1559.

Phillips, J.P. 1989, in IAU Symposium No. 191, Planetary Nebulae, ed. S. Torres-Peimbert (Dordrecht: Kluwer), p. 425.

Poulain, P. 1988, Astr. and Ap. Suppl., $72,215$.

Renzini, A. and Buzzoni, A. 1986 in Spectral Evolution of Galaxies, ed. C. Chiosi and A. Renzini (Dordrecht: Reidel), p. 195.

Saha, A. and Hoessel, J.G. 1990, A.J., 99, 97.

Sandage, A. and Tammann, G.A. 1981, A Revised Shapley-Ames Catalog of Bright Galaxies, (Washington: Carnegie Institute of Washington).

Schneider, S.E. and Terzian, Y. 1983, Ap. J., 274, L61.

Seaton, M.J. 1968, Ap. (Letters), 2, 55.

Weidemann, V. 1977, Astr. and Ap., 61, L27.

Weidemann, V. 1991, in Proc. $7^{\text {th }}$ European Workshop on White Dwarfs, eds. G. Vauclair and E.M. Sion (Dordrecht: Kluwer), p. 67. 